\title{
Outcomes in Adult Scoliosis Patients Who Undergo Spinal Fusion Stopping at L5 Compared with Extension to the Sacrum
}

\author{
Zeeshan M. Sardar $^{1}$ Jean A. Ouellet ${ }^{1}$ Dena J. Fischer ${ }^{2}$ Andrea C. Skelly ${ }^{2}$ \\ ${ }^{1}$ McGill Scoliosis and Spine Centre, McGill University Health Centre, \\ Address for correspondence Dr. Jean A. Ouellet, MD, FRCSC, Montreal \\ Montreal, Quebec, Canada \\ General Hospital, 1650 Cedar Avenue, T8-200, Montreal, QC, Canada \\ ${ }^{2}$ Spectrum Research, Inc., Tacoma, Washington, United States \\ H3G 1A4 (e-mail: jean.ouellet@muhc.mcgill.ca).
}

Evid Based Spine Care J 2013;4:96-104.

\begin{abstract}
Study Design Systematic review.

Study Rationale Adult scoliosis is a common disorder that is associated with significantly higher pain, functional impairment, and effect on quality of life than those without scoliosis. Surgical spinal fusion has led to quantifiable improvement in patient's quality of life. However, for patients undergoing long lumbar fusion, the decision to stop the fusion at $L 5$ or to extend to $\mathrm{S} 1$, particularly if the $\mathrm{L} 5-\mathrm{S} 1$ disc is healthy, remains controversial.

Objective The aim of the study is to evaluate if fusion stopping at L5 increases the comparative rates of revision, correction loss, and/or poor functional outcomes compared with extension to the sacrum in adult scoliosis patients who require spinal fusion surgery.

Materials and Methods A systematic review of the literature was performed using PubMed, the National Guideline Clearinghouse Database and bibliographies of key articles that evaluated adult scoliosis patients who required spinal fusion surgery and compared outcomes for fusions to the sacrum versus stopping at L5. Articles were included on the basis of predetermined criteria and were appraised using a predefined quality-rating scheme.

Results From 111 citations, 26 articles underwent full-text review, and 3 retrospective cohort studies met all inclusion and exclusion criteria. Revision rates in subjects who underwent spinal fusion to L5 (20.8-23.5\%) were lower in two studies compared with those with fusion extending to the sacrum (19.0-58.3\%). Studies that assessed

\section{Keywords}

- scoliosis

- spinal fusion

- sacrum

- lumbar

- adult deformity correction used different measures, making comparison across studies difficult. No significant differences were found in patient-reported functional outcomes across two studies that used different measures.

Conclusion The limited data available suggest that differences in revision rates did not consistently reach statistical significance across studies that compared spinal fusion to $\mathrm{L} 5$ versus extension to sacrum in adult scoliosis patients.
\end{abstract}

received

May 15, 2013

accepted after revision

July 18,2013 (c) 2013 Georg Thieme Verlag KG

Stuttgart · New York
DOI http://dx.doi.org/ 10.1055/s-0033-1357360. ISSN $1663-7976$. 


\section{Study Rationale and Context}

Adult scoliosis (Cobb angle $>10$ degrees) is a common disorder with reported prevalence up to 60 to $68 \% \%^{1,2}$ and appears to be more prevalent and more severe in women. ${ }^{1,3-5}$ The relationship between the magnitude of deformity and the severity and existence of symptoms has not been clearly established. ${ }^{1,5}$ However, adults with scoliosis report significantly higher pain, functional impairment, and effect on quality of life than those without scoliosis. ${ }^{1,5,6}$ In adults with scoliosis, sagittal balance has the most significant impact on pain, function, and progression of deformity compared with other radiographic parameters. ${ }^{7}$ The main goals of surgery are to achieve spinal balance, spinal stabilization, and neural decompression. For patients requiring long fusion into the lumbar spine with a relatively healthy L5-S1 motion segment in the absence of Spondylolisthesis, previous decompression, stenosis, or fixed obliquity of the L5-S1 motion segment, ${ }^{4,6,8-11}$ the decision to choose whether to stop the fusion at L5 or to extend to S1 remains controversial. ${ }^{8-11}$

\section{Objectives}

The aim of the study is to evaluate if fusion stopping at L5 increases the comparative rates of revision, correction loss, and/or poor functional outcomes compared with extension to the sacrum in adult scoliosis patients who require spinal fusion surgery.

\section{Materials and Methods}

Study design: Systematic review.

Search: The databases included PubMed and National Guideline Clearinghouse Databases, as well as bibliographies of key articles.

Dates searched: The dates were searched from 1950 to April 2013.

Inclusion criteria: The patient should be 18 years or older at the time of surgery; diagnosis of adult idiopathic scoliosis or adult degenerative scoliosis and fusion of three or more segments were included in the study.

Exclusion criteria: Neuromuscular scoliosis, fusion for traumatic disorders, prior fusion surgery, less than $80 \%$ of study population with diagnosis of adult idiopathic/degenerative scoliosis or meeting other inclusion criteria; studies with less than 10 subjects and case series were excluded from the study.

Prognostic factors: The prognostic factors include the following: primary factor-length of fusion (spinal fusion stopping at L5 vs. extension to sacrum); secondary factors (potentially confounding factors)-age, number of fused segments, type of fusion (anterior, posterior, and combined), and patient comorbidities.

Outcomes: The outcomes include rate of revision surgery, loss of deformity correction (lumbar lordosis, sagittal balance), and poor functional outcome scores.

Analysis: Descriptive statistics; statistics and effect estimates as reported by the respective authors of each study included in this review.
Overall strength of evidence: Risk of bias for individual studies was based on using criteria set by The Journal of Bone and Joint Surgery, ${ }^{12}$ modified to delineate criteria associated with methodological quality and risk of bias based on recommendation from the Agency for Healthcare Research and Quality (AHRQ). ${ }^{13,14}$ The overall strength evidence across studies was based on precepts outlined by the Grades of Recommendation Assessment, Development and Evaluation Working Group ${ }^{15}$ and recommendations made by the AHRQ. ${ }^{13,14}$

Details about methods can be found in the online supplementary material.

\section{Results}

- The search yielded 111 citations, 26 of which underwent full-text review (-Fig. 1).

- Three unique studies of adult scoliosis patients evaluating outcomes following spinal fusion to L5 compared with extension to the sacrum met the inclusion criteria.

- There was one moderate quality retrospective cohort study (Class of Evidence [CoE] II), ${ }^{16}$ and two poor-quality retrospective cohort studies ( $\mathrm{CoE}$ III). $)^{3,17}$

- In addition, details regarding the critical appraisal and study exclusion criteria can be found in the online supplementary material.

- -Table 1 describes the characteristics of included studies including subject and treatment characteristics. The mean length of follow-up for included studies was 3.5 to 4.8 (range, 2.0-14.3) years. The mean age in one study was almost 20 years older than the other studies. ${ }^{3}$ - Table 2 summarizes outcomes evaluated and effect size estimates, if reported in the studies.

\section{Revision}

- Revision rates were assessed in all studies. Differences between groups did not consistently reach statistical significance across studies (-Table 2, - Fig. 2 ).

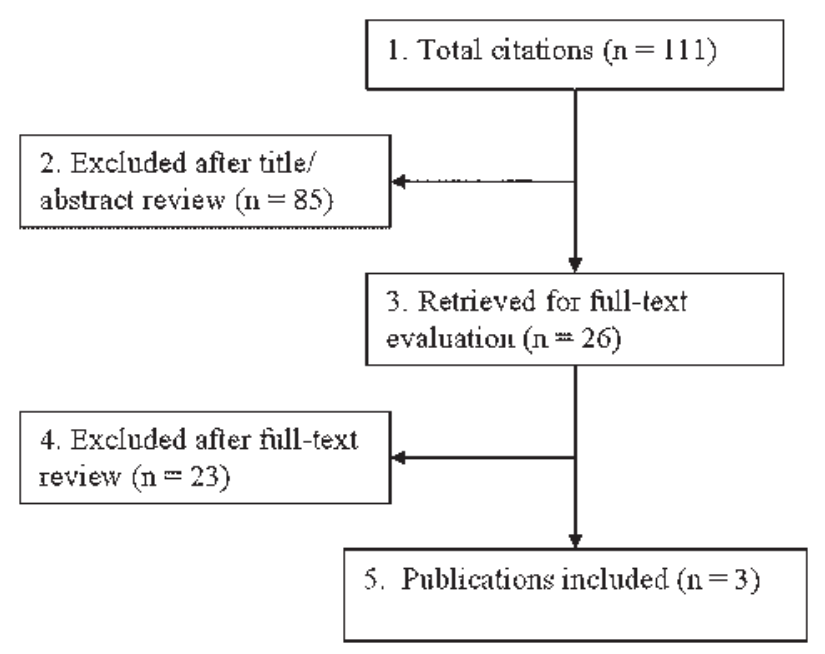

Fig. 1 Flowchart showing results of literature search. 


\begin{tabular}{|c|c|c|c|}
\hline 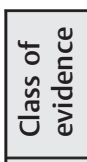 & $=$ & $\equiv$ & $\equiv$ \\
\hline 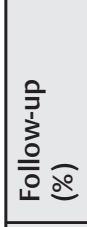 & 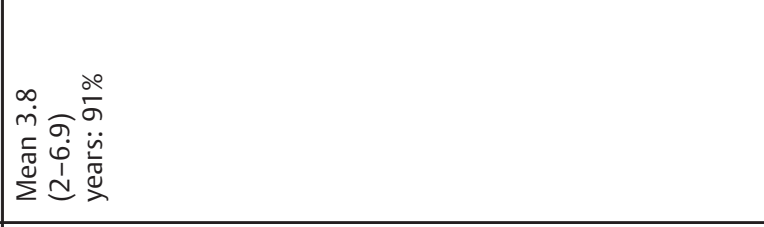 & 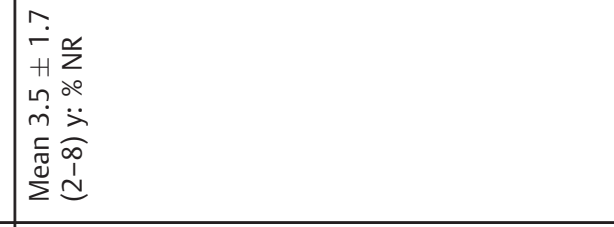 & 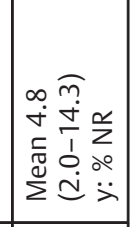 \\
\hline 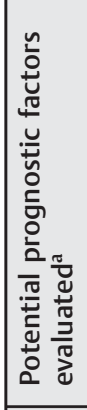 & 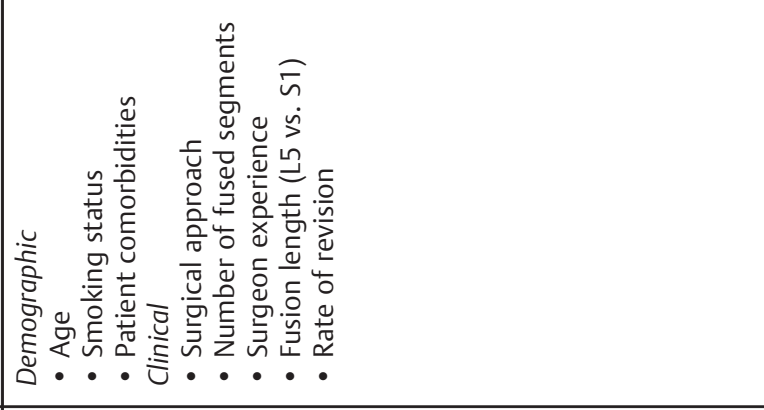 & 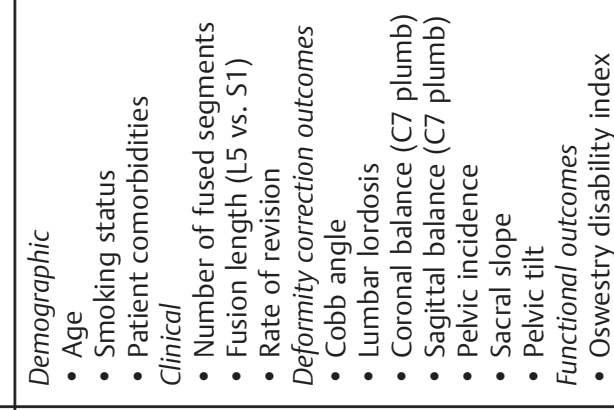 & 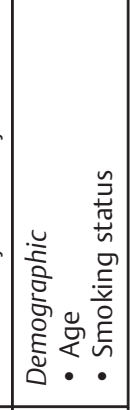 \\
\hline & 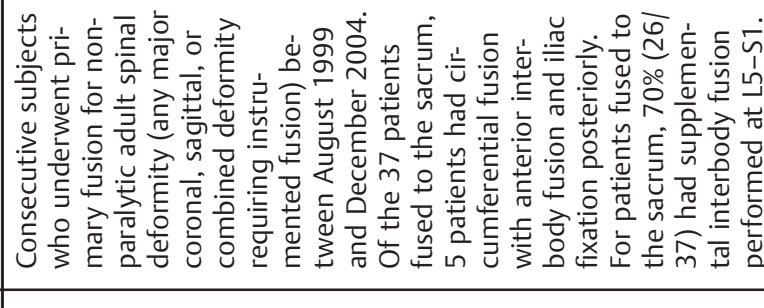 & 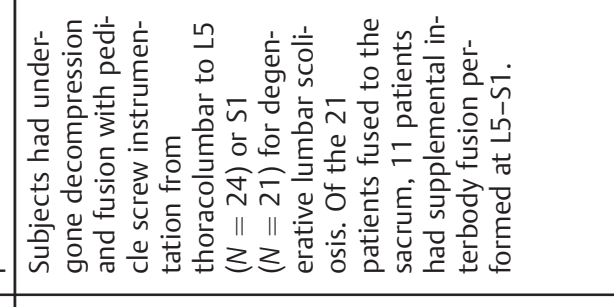 & 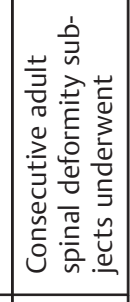 \\
\hline & 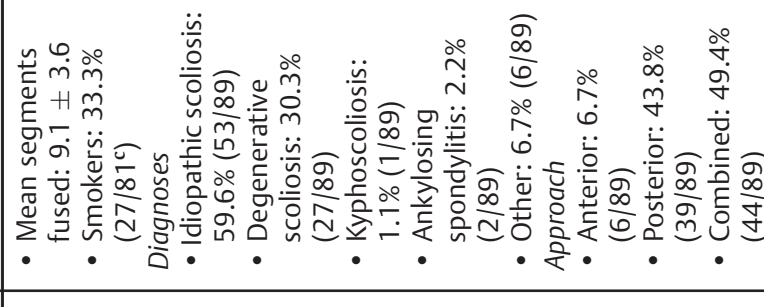 & 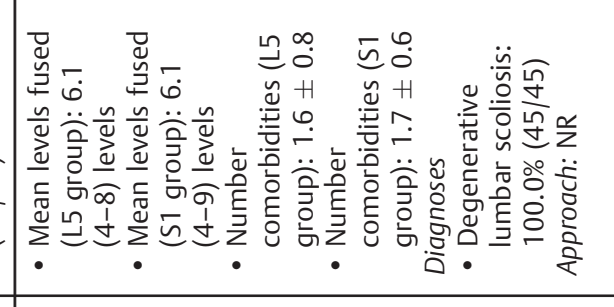 & 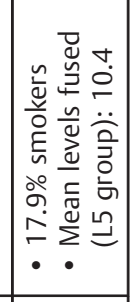 \\
\hline 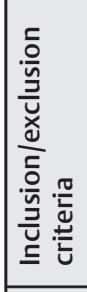 & 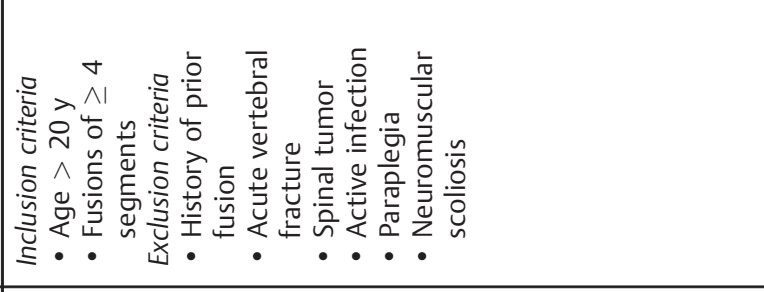 & 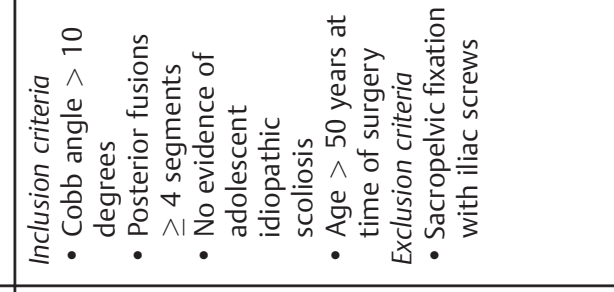 & 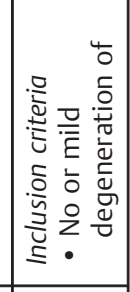 \\
\hline 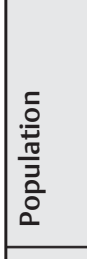 & 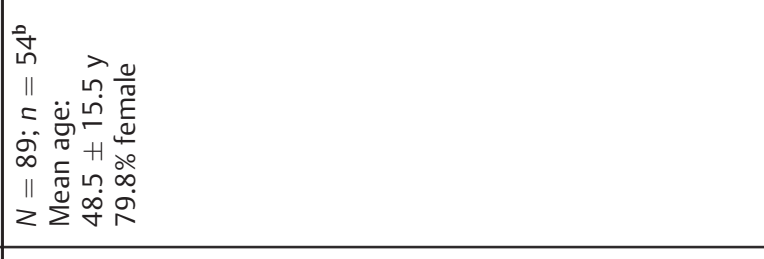 & 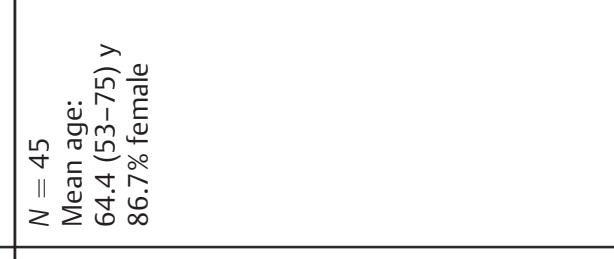 & 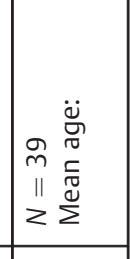 \\
\hline 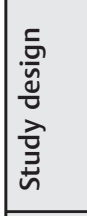 & 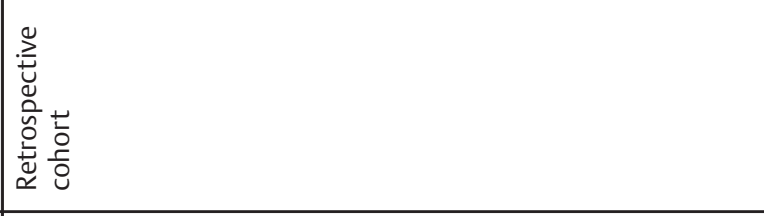 & 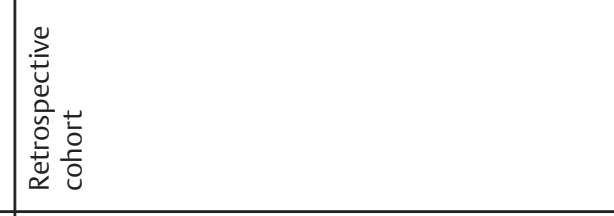 & 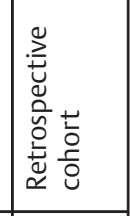 \\
\hline$\geqq$ & 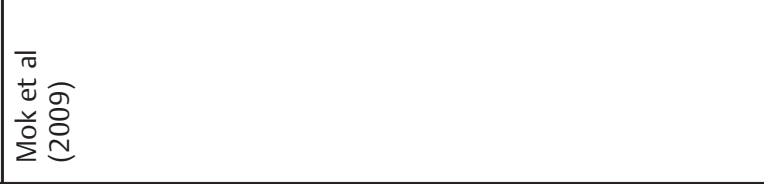 & 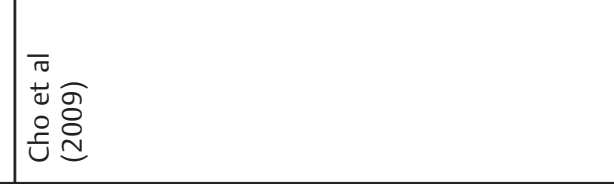 & 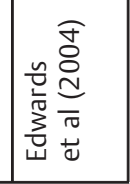 \\
\hline
\end{tabular}



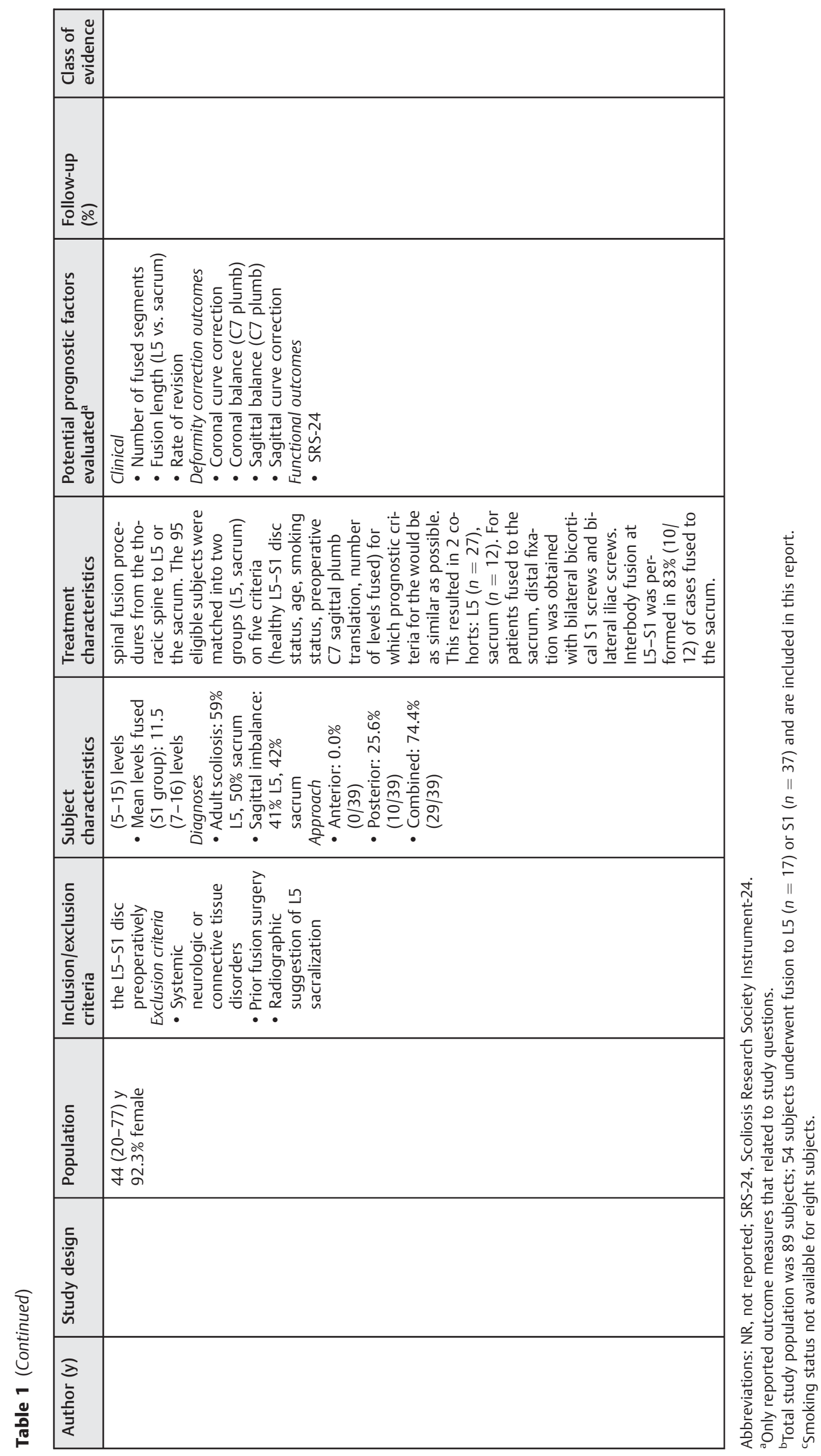


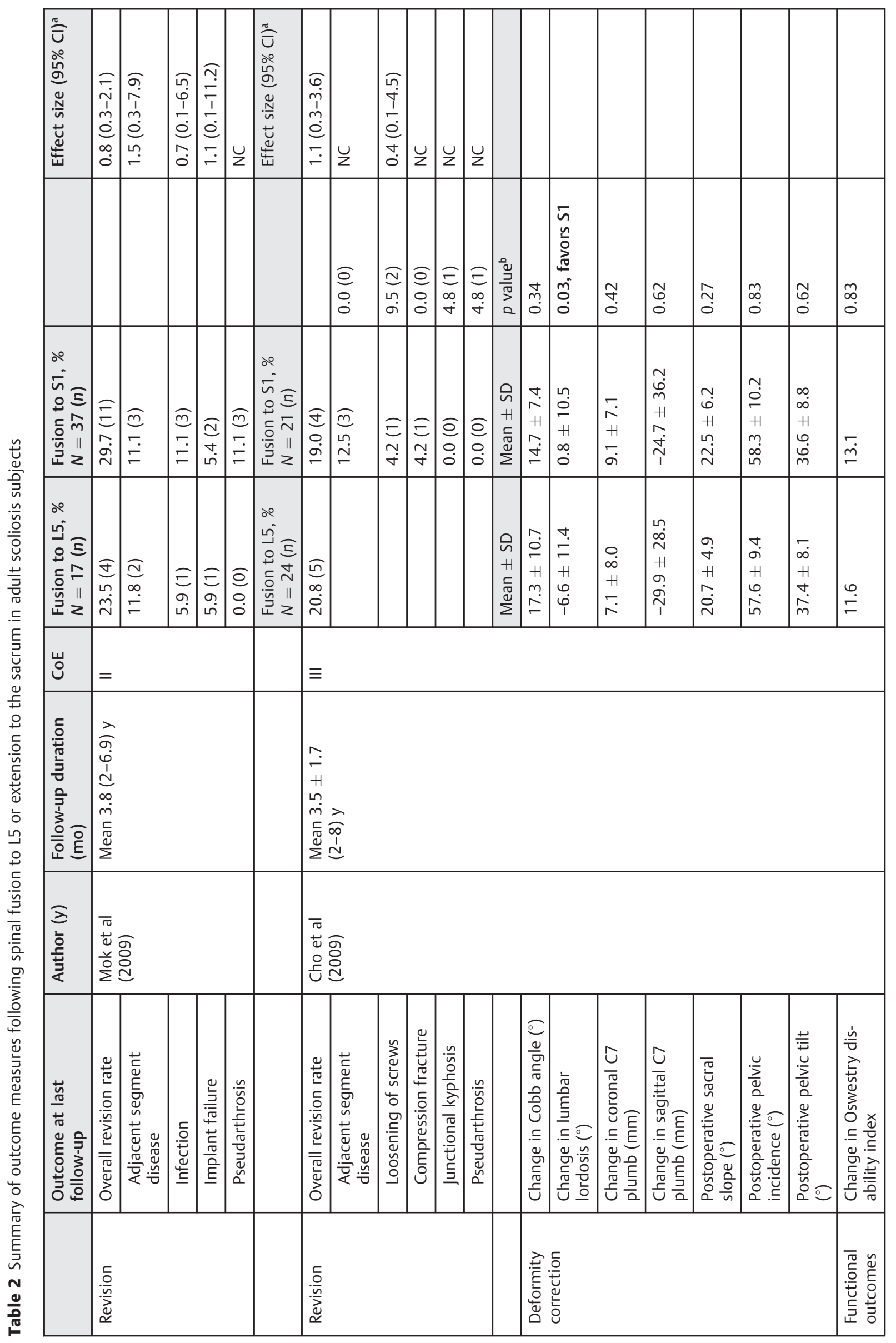




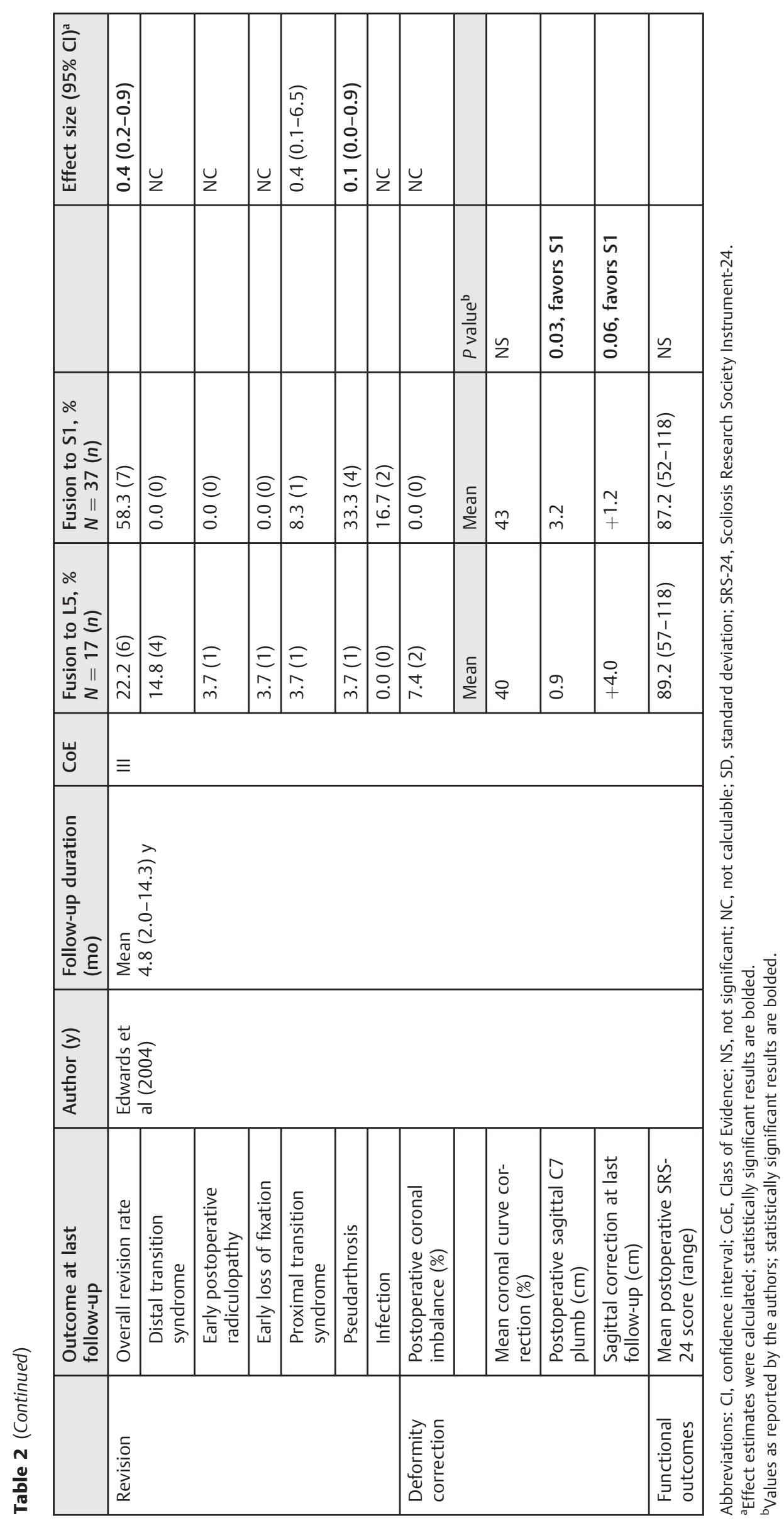




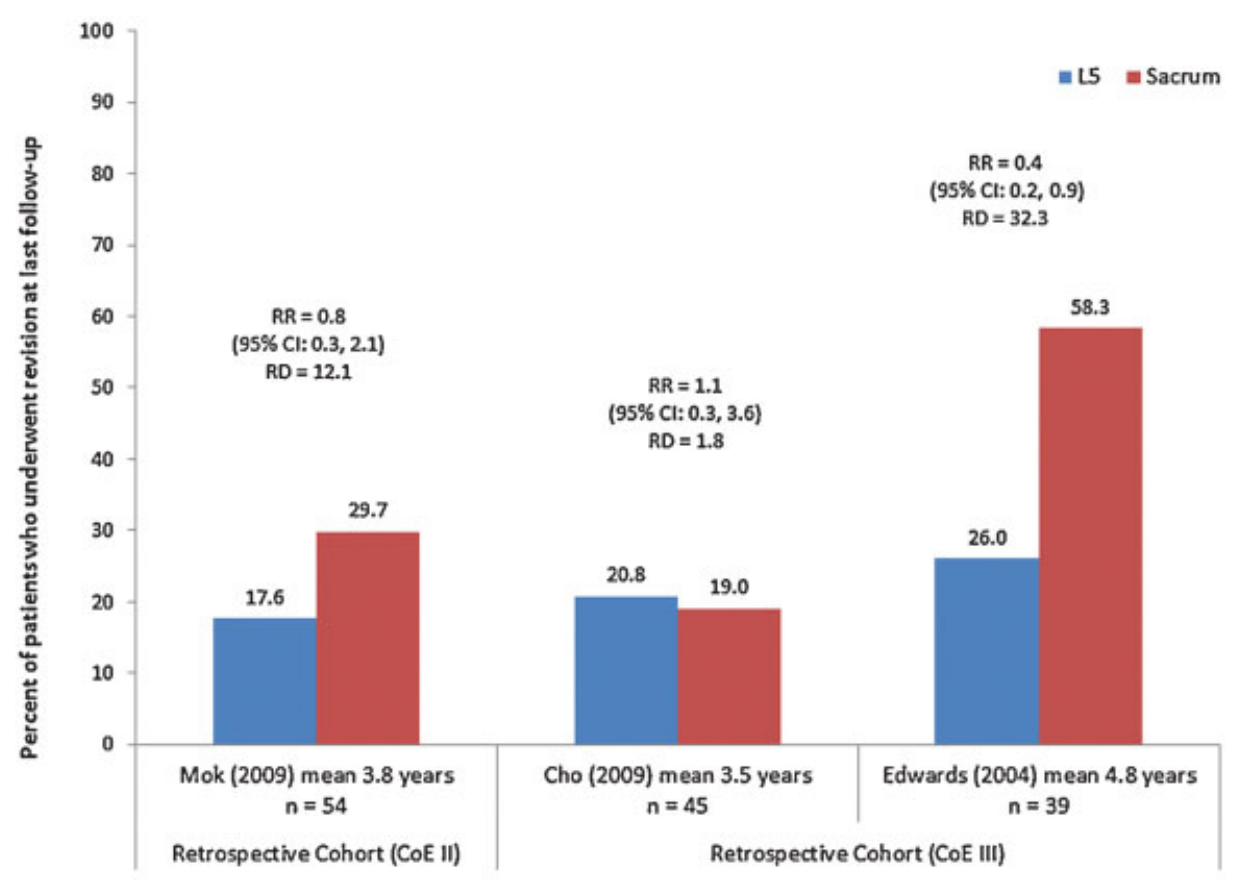

Fig. 2 Revision rates in adult scoliosis patients who underwent spinal fusion to L5 compared with S1.

- Revision rates among subjects with fusion to L5 (20.823.5\%) were lower in two studies compared with those who underwent extension to the sacrum (19.0-58.3\%).

- Revision due to pseudarthrosis was more frequent for those whose fusions extended to the sacrum $(4.8-33.3 \%)$ across all studies compared with those having fusion to L5 (0.0-3.7\%). Small sample sizes may preclude effective evaluation of statistical differences.

- Findings within individual studies:

- A CoE II retrospective cohort study of adult spinal deformity subjects found a lower but nonstatistically significant difference in revision rates between the L5 and $\mathrm{S} 1$ fusion groups ( $\mathrm{L} 5=23.5 \%, \mathrm{~S} 1=29.7 \%$; RR 0.8 , 95\% CI: 0.3-2.1). ${ }^{16}$

- One CoE III retrospective cohort study of degenerative lumbar scoliosis, subjects older than 50 years at the time of surgery found no significant differences in revision rates between the $\mathrm{L} 5$ and $\mathrm{S} 1$ fusion groups ( L5 = 20.8\%, S1 = 19.0\%; RR 1.1, 95\% CI: 0.3-3.6). ${ }^{3}$

- In another CoE III retrospective cohort study of adult spinal deformity subjects who were matched into two cohorts (fusion to L5, fusion to sacrum) based upon five criteria (healthy L5-S1 disc status, age, smoking status, preoperative $\mathrm{C} 7$ sagittal plumb translation, and number of levels fused), revision rates were significantly lower in the fusion to L5 compared with fusion to sacrum group $(\mathrm{L} 5=22.2 \%$, sacrum $=58.3 \%$; RR 0.4 , 95\% CI: 0.2-0.9). ${ }^{17}$

\section{Deformity Correction}

- Deformity correction was evaluated in two CoE III retrospective cohort studies. Each used different measures, making comparison across studies difficult.
- One study of degenerative lumbar scoliosis subjects older than 50 years at the time of surgery found that lumbar lordosis was significantly improved at last follow-up in the S1 compared with L5 fusion groups $(p=0.03)$. There were no statistically significant differences between groups in other deformity correction measures: Cobb angle, coronal balance, sagittal balance, pelvic incidence, sacral slope, or pelvic tilt. ${ }^{3}$ - The study of adult spinal deformity subjects who were matched into two cohorts reported a statistically significant improvement in sagittal balance $(p=0.03)$, and sagittal correction was marginally significant $(p=0.06)$, favoring the sacrum compared with L5 group. There were no significant differences in coronal balance or coronal curve correction measures between groups. ${ }^{17}$

\section{Functional (Patient-Reported) Outcomes}

- There were no significant differences between groups in functional outcomes across two studies using different measures.

- In the study of degenerative lumbar scoliosis subjects older than 50 years at the time of surgery, there were no differences between groups in Oswestry disability index score changes between preoperative and last follow-up. ${ }^{3}$

- The study of adult spinal deformity subjects who were matched into two cohorts found no differences in mean postoperative Scoliosis Research Society-24 (SRS-24) scores. ${ }^{17}$

\section{Clinical Guidelines}

No relevant clinical guidelines were identified. 
Table 3 Evidence summary

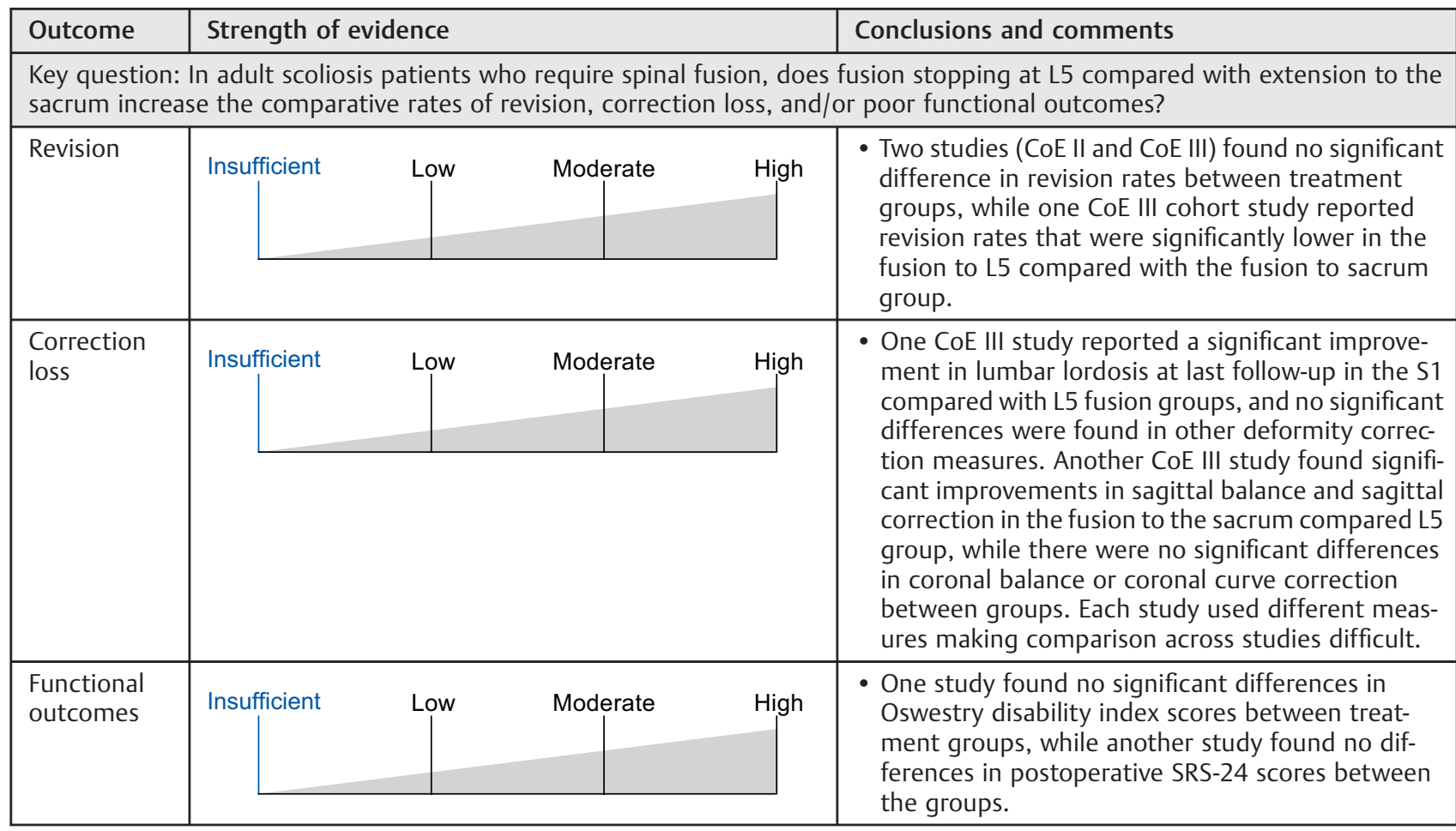

Abbreviations: CoE, Class of Evidence; SRS-24, Scoliosis Research Society Instrument-24.

\section{Evidence Summary}

- In adult scoliosis patients who underwent spinal fusion to L5 compared with extension to the sacrum, the overall strength of evidence was graded as insufficient for drawing conclusions regarding comparative rates of revision, correction loss, and functional outcomes (- Table $\mathbf{3}$ ). We have very little confidence in the effect estimate: The true effect is likely to be substantially different than the estimated effect.

\section{Discussion}

- Although there is limited data available, differences in revision rates between groups did not consistently reach statistical significance across studies. Studies that assessed deformity correction used different measures, making comparison across studies difficult. There were no significant differences in functional outcomes across two studies that used different measures.

- However, contrary to clinical suspicion, none of the studies showed a higher revision rate if the fusion was stopped at L5 instead of extending the fusion to S1. It is more important to note that both the treatment groups were associated with a high revision rate.

- Conclusions from this systematic review are limited by the paucity of high-quality studies that assessed the comparisons of interest. Furthermore, because of small sample sizes, studies may not have been sufficiently powered to detect differences between treatment groups, particularly for rare outcomes. An additional limitation included variability in outcome measures used to evaluate deformity correction across studies.

- Considering these unequivocal results, we emphasize the importance of involving the patient in the decision-making process and making them aware of the risk of revision surgery regardless of whether the fusion is stopped at L5 or extended to S1.

- Future research should be aimed at high-quality studies that prospectively assess the comparators of interest, with sufficient sample size to detect differences between treatment groups. Furthermore, spinal construct survivorship should be studied and factors influencing survivorship could be a better way to attempt to answer this clinical dilemma.

\section{Acknowledgment}

Analytic support for this work was provided by Spectrum Research, Inc. with funding from AOSpine.

\section{Disclosures}

Jean Ouellet received no funding pertaining to this article. Funding not related to the current work includes the following: Consulting, Synthes; Teaching, AO Foundation; Fellowship support, AO Foundation; and Research grants, AO Foundation. Zeeshan Sardar has no conflicts of interest or funding sources to disclose. 


\section{References}

1 Kilshaw M, Baker RP, Gardner R, Charosky S, Harding I. Abnormalities of the lumbar spine in the coronal plane on plain abdominal radiographs. Eur Spine J 2011;20(3):429-433

2 Schwab F, el-Fegoun AB, Gamez L, Goodman H, Farcy JP. A lumbar classification of scoliosis in the adult patient: preliminary approach. Spine (Phila Pa 1976) 2005;30(14):1670-1673

3 Cho KJ, Suk SI, Park SR, et al. Arthrodesis to L5 versus S1 in long instrumentation and fusion for degenerative lumbar scoliosis. Eur Spine J 2009;18(4):531-537

4 Ohtori S, Sainoh T, Takaso M, et al. Clinical incidence of sacroiliac joint arthritis and pain after sacropelvic fixation for spinal deformity. Yonsei Med J 2012;53(2):416-421

5 Urrutia J, Espinosa J, Diaz-Ledezma C, Cabello C. The impact of lumbar scoliosis on pain, function and health-related quality of life in postmenopausal women. Eur Spine J 2011;20(12):2223-2227

6 Kebaish KM, Neubauer PR, Voros GD, Khoshnevisan MA, Skolasky RL. Scoliosis in adults aged forty years and older: prevalence and relationship to age, race, and gender. Spine (Phila Pa 1976) 2011; 36(9):731-736

7 Kim YJ, Bridwell KH, Lenke LG, Rhim S, Cheh G. Sagittal thoracic decompensation following long adult lumbar spinal instrumentation and fusion to L5 or S1: causes, prevalence, and risk factor analysis. Spine (Phila Pa 1976) 2006;31(20):2359-2366

8 Emami A, Deviren V, Berven S, Smith JA, Hu SS, Bradford DS. Outcome and complications of long fusions to the sacrum in adult spine deformity: luque-galveston, combined iliac and sacral screws, and sacral fixation. Spine (Phila Pa 1976) 2002;27(7): 776-786

9 Kostuik JP, Hall BB. Spinal fusions to the sacrum in adults with scoliosis. Spine (Phila Pa 1976) 1983;8(5):489-500

\section{Editorial Perspective}

The authors received praise from our reviewers for their clinically relevant topic selection. Although limited by a small number of publications, the authors could not find any difference in the (relatively high) revision rates of patients where a caudal fusion stopped at the sacrum or at L5, provided this was a healthy motion segment. Our reviewers pointed out some significant treatment variables, which had not been addressed in the source materials of the included studies.

1. Does the type of L5-S1 arthrodesis, instrumentation to the ilium, and possible addition of rhBMP-2, possibly lower the nonunion rates in patients fused to the sacrum?
10 Kuhns CA, Bridwell KH, Lenke LG, et al. Thoracolumbar deformity arthrodesis stopping at L5: fate of the L5-S1 disc, minimum 5-year follow-up. Spine (Phila Pa 1976) 2007;32(24):2771-2776

11 Weistroffer JK, Perra JH, Lonstein JE, et al. Complications in long fusions to the sacrum for adult scoliosis: minimum five-year analysis of fifty patients. Spine (Phila Pa 1976) 2008;33(13): $1478-1483$

12 Wright JG, Swiontkowski MF, Heckman JD. Introducing levels of evidence to the journal. J Bone Joint Surg Am 2003;85-A(1):1-3

13 Methods Guide for Effectiveness and Comparative Effectiveness Reviews. Rockville, MD: Agency for Healthcare Research and Quality; 2012

14 West S, King V, Carey TS, et al. Systems to Rate the Strength of Scientific Evidence. Evidence Report/Technology Assessment No. 47 (Prepared by the Research Triangle Institute-University of North Carolina Evidence-based Practice Center, Contract No. 290-97-0011). Rockville, MD: Agency for Healthcare Research and Quality; 2002

15 Atkins D, Best D, Briss PA, et al; GRADE Working Group. Grading quality of evidence and strength of recommendations. BMJ 2004; 328(7454):1490

16 Mok JM, Cloyd JM, Bradford DS, et al. Reoperation after primary fusion for adult spinal deformity: rate, reason, and timing. Spine (Phila Pa 1976) 2009;34(8):832-839

17 Edwards CC II, Bridwell KH, Patel A, Rinella AS, Berra A, Lenke LG. Long adult deformity fusions to L5 and the sacrum. A matched cohort analysis. Spine (Phila Pa 1976) 2004;29(18):19962005

2. Does age, gender, body mass, and restoration of sagittal balance at the time of the fusion influence the outcome of patients with caudal fusion ending at L5?

The authors of this systematic review have shown vividly that, by asking a simple question with a somewhat counterintuitive null-hypothesis, somewhat surprising results may emerge from a formal, well-performed, and objectively based systematic review such as was done here. This, in turn, challenges us to take the next steps in studying these findings further and looking to find explanations from longer term survivorship analyses and by taking some of the variables mentioned into consideration when planning future comparison studies. 